\title{
LEVERAGING MOTOR LEARNING FOR A TANGIBLE PASSWORD SYSTEM
}

\author{
Martez Edward Mott
}

\author{
A Thesis \\ Submitted to the Graduate College of Bowling Green \\ State University in partial fulfillment of \\ the requirements for the degree of
}

\section{MASTER OF SCIENCE}

May 2012

Committee:

Dr. G. Michael Poor, Advisor

Dr. Laura Leventhal

Dr. Guy Zimmerman 
(C) 2012

Martez E. Mott

All Rights Reserved 


\begin{abstract}
Dr. G. Michael Poor, Advisor

Tangible user interfaces (TUIs) may allow users to have more direct interaction with systems when compared to traditional graphical user interfaces (GUIs). However, the full range of applications where TUIs can be utilized in practice is unclear. To resolve this problem, the benefits of TUIs must be analyzed and matched to an application domain where they hold advantages over more traditional systems. Since TUIs require users to use their hands in order to interact with the system, there is the possibility for these systems to leverage motor leaning to perform specific tasks.

This work explored how motor learning can be used to create a tangible password system. A novel tangible password system was created and a small study conducted in order to identify future research objectives. Results show that users were successful in using the system to input their password and were able to recall their password after being removed from the system for a brief period of time.
\end{abstract}


To Nisha, we miss you. 


\section{ACKNOWLEDGMENTS}

I would first like to thank my committee for guiding me through this process. Not only did they help me through the process of completing my thesis, they played a vital role in encouraging me to pursue a graduate degree in Computer Science. I would also like to thank my lab mates for our great research conversations and our extremely random discussions on a wide array of topics. In particular, I would like to thank Thomas Donahue for his invaluable help on forming and reforming research ideas, bust most importantly, for his friendship.

I would finally like to thank my family and friends who have supported me during my entire academic career. I would especially like to thank Nona Ingram for her unwavering love and support. 


\section{Table of Contents}

$\begin{array}{lr}\text { CHAPTER 1: Introduction } & 1\end{array}$

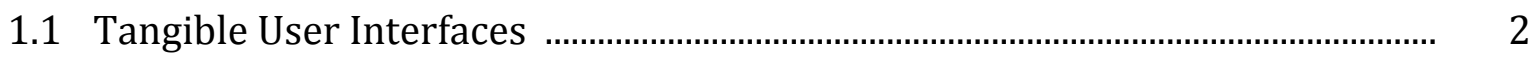

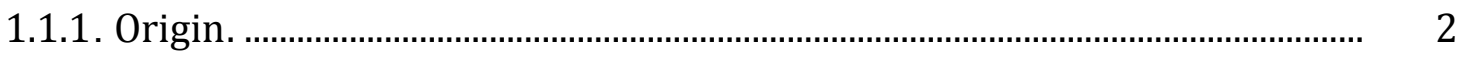

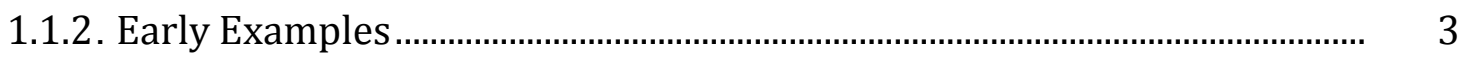

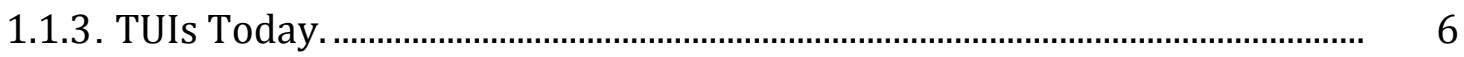

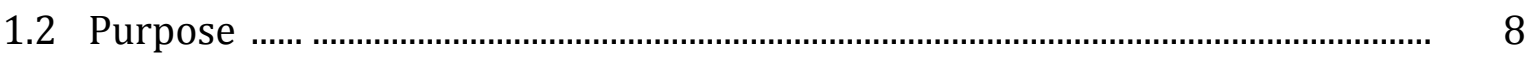

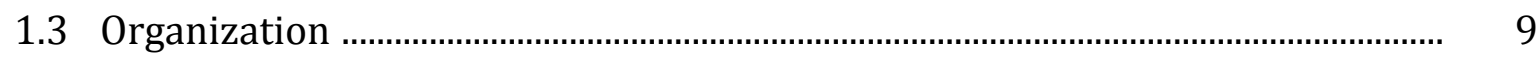

$\begin{array}{ll}\text { CHAPTER 2: Related Work } & 10\end{array}$

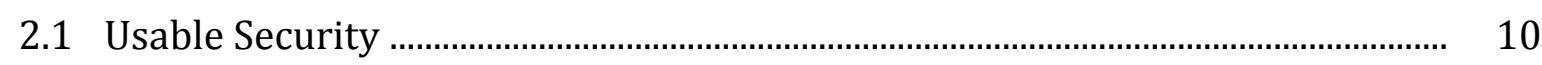

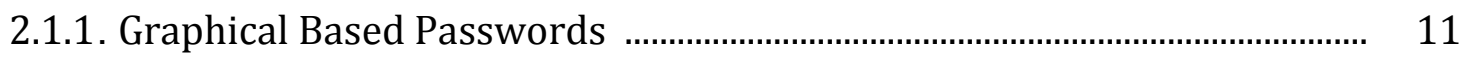

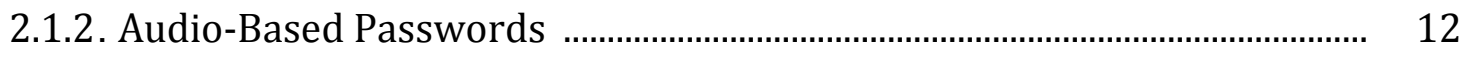

2.1.3. Icon-Based Passwords ................................................................................ 12

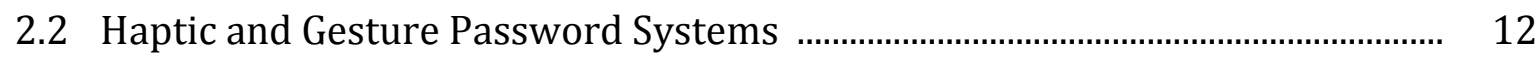

2.2.1. Haptic Password Systems ......................................................................... 13

2.2.2. Gesture Based Passwords ........................................................................... 14

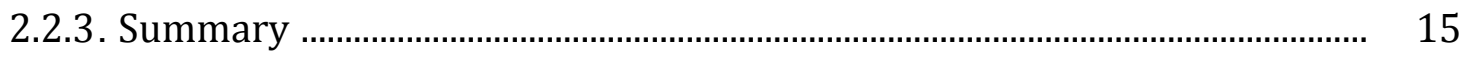

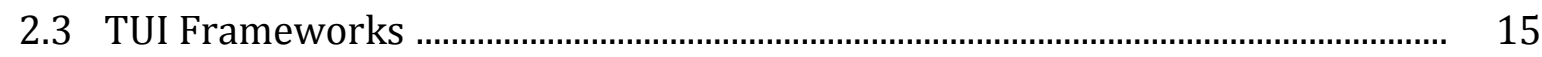


2.3.1. Fishkin Taxonomy …................................................................................... 15

2.3.2. The TAC Paradigm ....................................................................................... 17

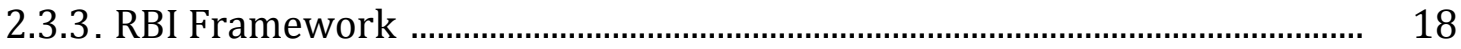

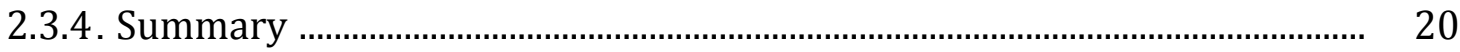

$\begin{array}{ll}\text { CHAPTER 3: TangibleRubik } & 21\end{array}$

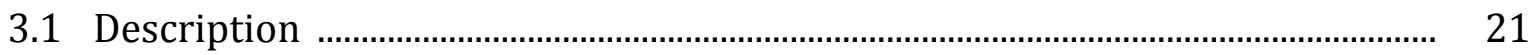

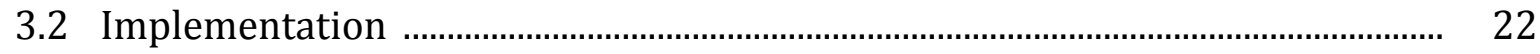

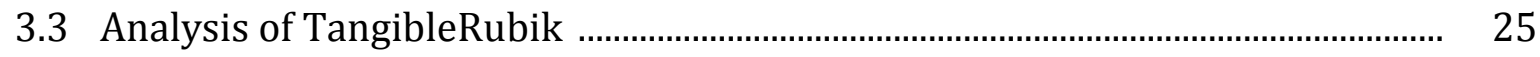

3.3.1. TangibleRubik and the Fishkin Taxonomy …............................................. 25

3.3.2. TangibleRubik and TAC ............................................................................ 26

3.3.3. TangibleRubik and RBI ............................................................................. 27

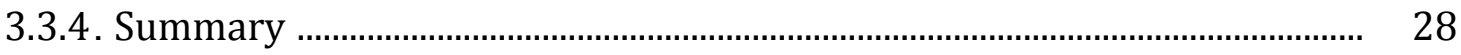

$\begin{array}{lr}\text { CHAPTER 4: Method } & 29\end{array}$

4.1 Introduction and Predictions ................................................................................ 29

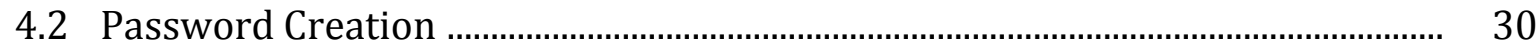

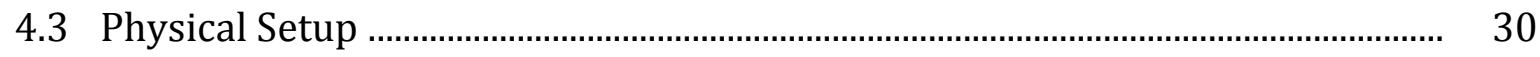

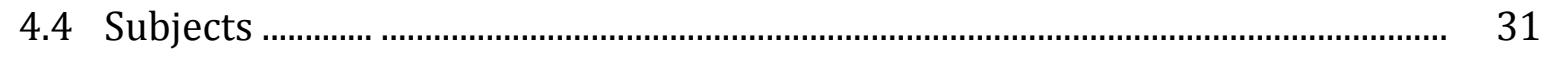

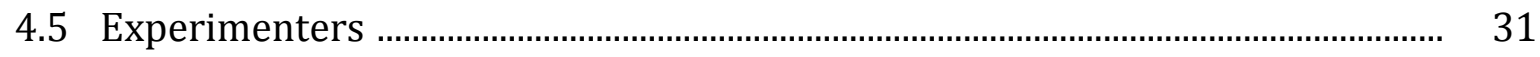

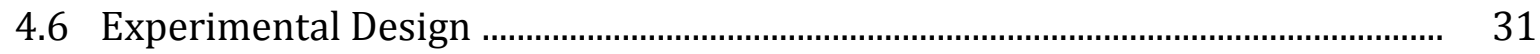

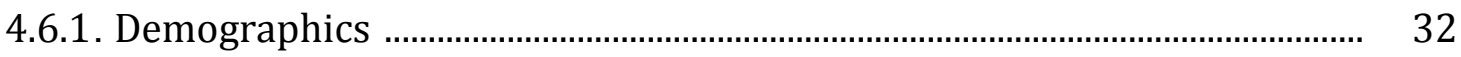

4.6.2. Practice and Experimental Trials ................................................................ 32

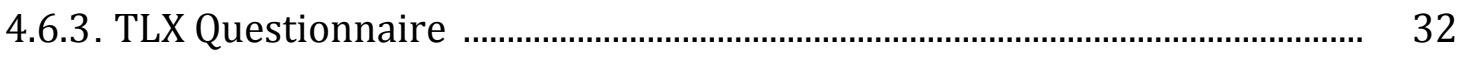

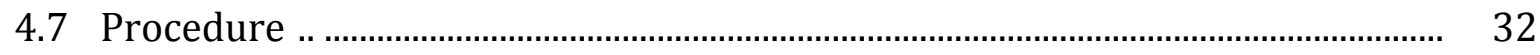




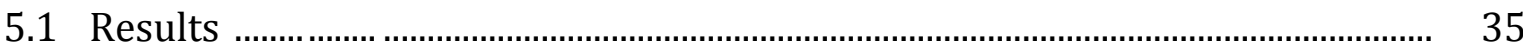

5.1.1. Impact of password lengths .......................................................................... 35

5.1.2. TLX Questionnaire ...................................................................................... 37

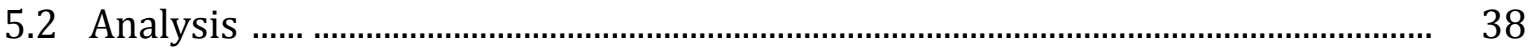

5.2.1. Analysis of Error Rates ....................................................................................... 38

5.2.2. Analysis of Password Entry Times .............................................................. 38

CHAPTER 6: Discussion and Conclusion $\quad 39$

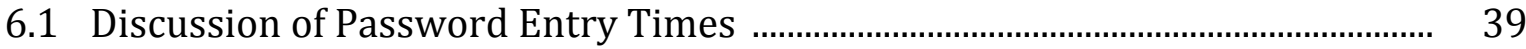

6.2 Discussion of Password Memorability ……………................................................... 40

6.3 Discussion of Password Length ............................................................................ 41

6.4 TangibleRubik and Password-Composition Policies................................................ 41

6.5 User Created Passwords........................................................................................ 42

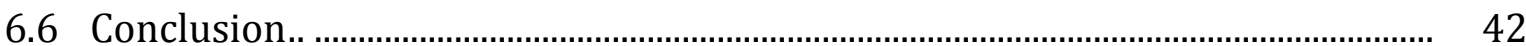

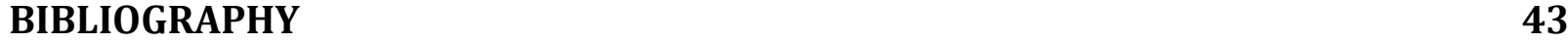




\section{List of Figures}

1.1 Ishii's representation of the transition from a GUI to a TUI .................................. 3

1.2 Illustration of Bishop's Marble Answering Machine .............................................. 4

1.3 Illustration of the linking of a brick with a virtual object...................................... 5

1.4 Illustration showing how altering the physical handle manipulate the Underlying virtual object

5

1.5 Two bricks used to alter the size of the virtual object (left). A pictorial Representation of a user performing a pinch gesture (right) …….....................

3.1 Simple GUI used for scanning during training ................................................... 23

3.2 TangibleRubik, in orientation held by participants, identifying the faces as in

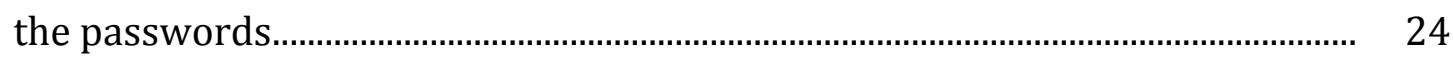

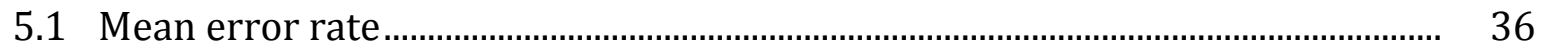

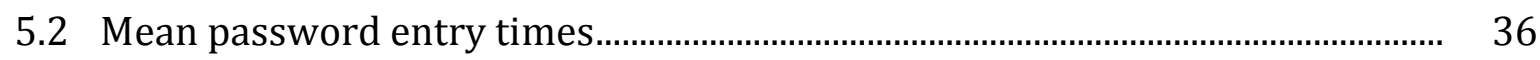

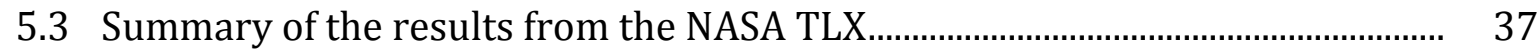




\section{CHAPTER 1}

\section{Introduction}

Computing systems are pervasive in nearly every activity of day-to-day life, varying greatly in size, location, and functionality. This versatility allows people to carry small portable computing devices for entertainment and communication while away from their homes and offices. In public places, ATMs and self-serving kiosks allow individuals to receive information quickly and avoid long lines at banks, grocery stores, and movie theatres. With the growing number and availability of computing systems, user interface design will most likely continue to be one of the most discussed topics in the Human-Computer Interaction (HCI) community. Since user interface design is one the main focuses in HCI, researchers are constantly searching for new ways for users to interact with computing systems. While researchers and practitioners still attempt to improve the usability of command-line and graphical user interfaces (CLIs and GUIs), tangible user interfaces (TUIs) have received a lot of attention from the HCI community over the years. TUIs are relatively unknown to the public, but they can provide a host of new and exciting interaction styles. 


\subsection{Tangible User Interfaces}

\subsubsection{Origin}

The concept of a tangible user interface was first proposed by Hiroshi Ishii and Brygg Ullmer in 1997 in their visionary Tangible Bits paper. In it, they describe Tangible Bits as "the coupling of bits with everyday physical objects and architectural surfaces [14]." Their work was motivated by the lack of physical objects with "rich affordances" in classrooms and laboratories. They claim the type of physical tools our ancestors used for performing calculations and collecting measurements have been replaced by digital technologies that do not offer the same physical richness. As a result, they proposed a way to merge the physical and digital worlds. By doing so, they hoped to improve the quality and number of interactions between people and digital information. This led to the creation of the term "tangible user interface" as any user interface (UI) that "augments the real physical world by coupling digital information to everyday physical objects and environments".

This type of interaction provided a drastic change from the more traditional WIMP (windows, icons, menus, and pointing device) interaction style, which lacks diversity with their input and output devices. Commonly, desktop GUIs rely on a mouse/keyboard for input and a monitor/speaker/printer for output. Compare this to the TUI concept where any real-world object can be used as an interface to its digital counterpart (see figure 1.1). TUIs provide a host of new possibilities, as the interaction is limited only by our senses, and what is available in the physical world. This allows users to have a richer experience with digital information. 


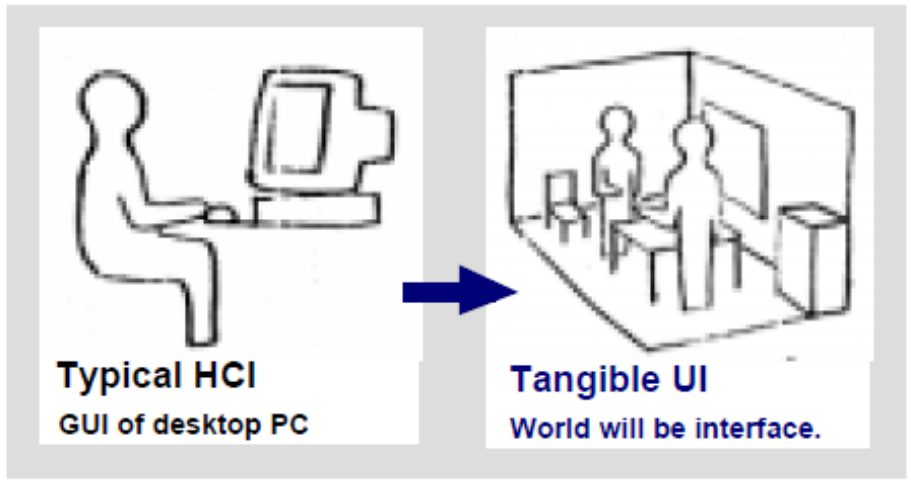

Figure 1.1: Ishii's representation of the transition from a GUI to a TUI [14]

\subsubsection{Early Examples}

Although the official definition of a TUI was not coined until 1997, some earlier works exhibit many of the same attributes. One of the most well-known is the Marble Answering Machine created by Durrell Bishop during his time at the Royal College of Art (see figure 1.2) [26]. 


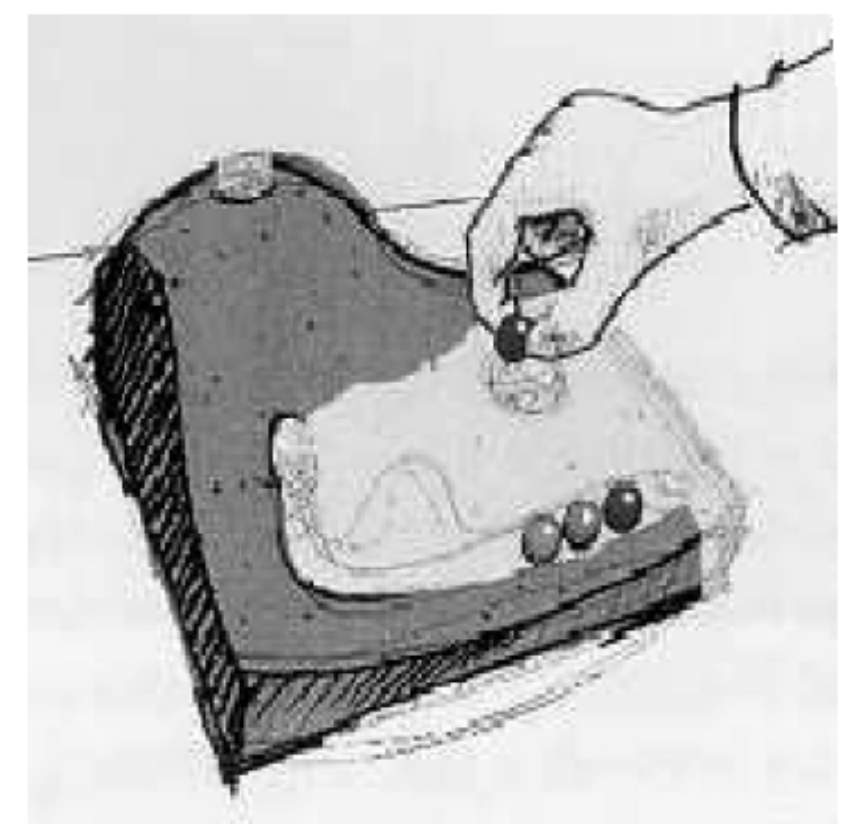

Figure 1.2: Illustration of Bishop's Marble Answering Machine [26]

In Bishop's prototype, messages are instantiated on a physical marble. A user could then play the message by physically grasping the marble, picking it up, and placing it in an indentation. The user could also redial the caller by placing the marble in an indentation near an augmented telephone. This prototype provided a clear and innovative demonstration of how developers can link digital information to a real-world object.

A second example, and a direct ancestor of the TUI, is Graspable User Interfaces. Graspable user interfaces are interfaces where the user has direct control of a virtual object through a physical handle. Bricks are an example of a graspable user interface where a physical object (a brick) is directly coupled with an underlying virtual object [10]. When a user places a brick onto a surface, on top of a virtual object, the two objects become linked; 
with the brick now acting as a physical handle (see Figure 1.3). By physically altering the brick a user is able to manipulate the underlying virtual object (see Figure 1.4).

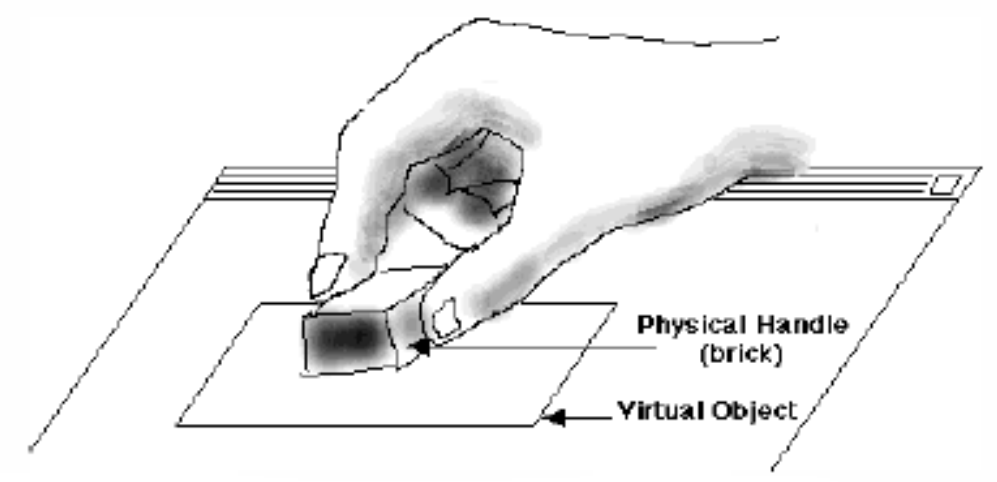

Figure 1.3: Illustration of the linking of a brick with a virtual object [10]

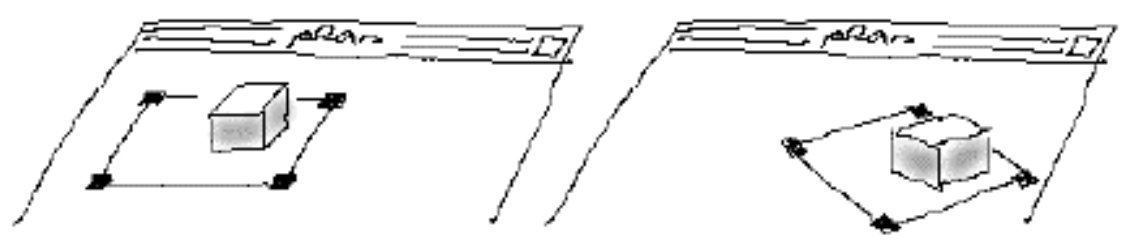

Figure 1.4: Illustration showing how altering the physical handle manipulates the underlying virtual object [10]

The bricks paradigm presented two important concepts that were later used in the creation and conceptualization of future TUIs. The first is the ability for space-multiplexed input. Space-multiplexed input allows multiple objects to occupy different spaces and to have different functions. Brick's accomplishes this by allowing users to have multiple physical handles present at a given time. In contrast, time-multiplexed input allows only one 
object to control different functions. In a traditional GUI there is a disconnect as the graphical output is often space-multiplexed (buttons, frames, etc) while the input is timemultiplexed (e.g. a mouse).

\subsubsection{TUIs Today}

A great majority of TUIs in use today are only available inside research laboratories [7]. This is because many TUI setups require specialized hardware and software that are not readily available to the public. For example, a collaborative virtual environment TUI developed by researchers at Georgia Institute of Technology required infrared pens, an infrared camera, a top-down projector and a Tangible Tracking Table [32]. Another TUI, created at the Massachusetts Institute of Technology Media Lab, uses over 200 boxes (3" x 3 " x 1.5") capable of emitting a variety of different colored lights [6]. Both examples demonstrate the portability and scalability issues concerning many TUIs created at research institutions.

Even with the issues TUIs face with being available for general use, researchers have been persistent in their attempt to utilize TUIs in many different areas. Some of the most intriguing are TUIs for learning, programming, music, entertainment, and edutainment.

Although the public may not get the opportunity to experience all of the TUIs found in research labs around the world, many popular commercial devices utilize basic TUI concepts, such as gesture-based interaction. The most prevalent examples are touchscreen smartphones and tablets. Since one of the main emphases of TUIs is hands-on physical 
manipulation, many popular smartphones employ these methods with gestures. One of the most popular is the swipe gesture. A swipe gesture allows users to easily perform task such as view of series of pictures on a mobile device by swiping their finger from one side of the touchscreen to the other (e.g. bottom to top or left to right). By doing so, the user is manipulating the digital information (the digital pictures) as if he or she is flipping through an actual photo album.

Another popular gesture is the pinch gesture. The pinch gesture allows a user to use two fingers to zoom in (move fingers closer together) or zoom out (move fingers farther apart) on an object on the screen. This gesture allows the user to manipulate the object on the screen as if it were on a malleable piece of paper. The pinch could then represent either the stretching or the shrinking of the paper. This multi-touch interaction is similar to the bricks paradigm discussed previously (see Figure 1.5).

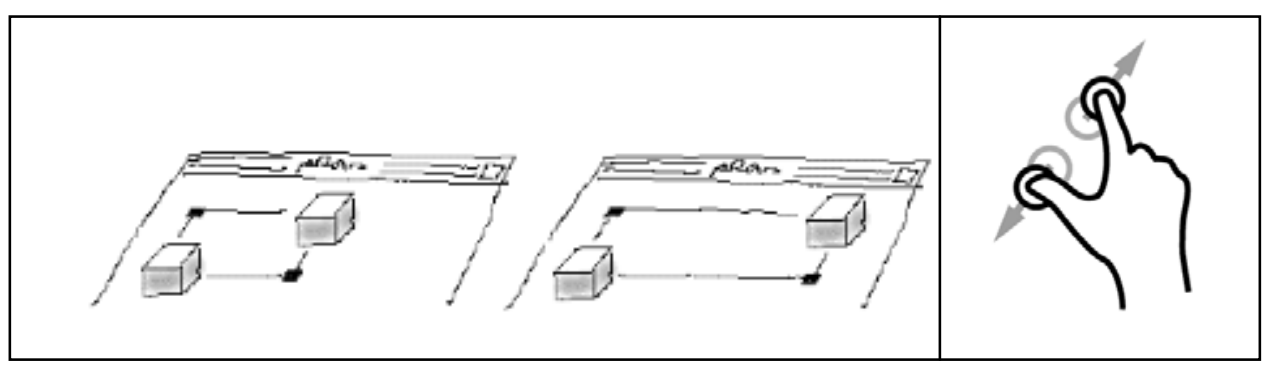

Figure 1.5: Two bricks used to alter the size of the virtual object (left). A pictorial representation of a user performing a pinch gesture (right) 


\subsection{Purpose}

With the popularity of TUIs growing rapidly, and similar interaction techniques becoming an important component of many devices (smartphones, etc), there is the need to develop and test new TUIs to discover their benefits over more traditional systems. Attempts have been made by the research community to develop tools and frameworks to aid in the development, implementation and evaluation of TUIs [15] [24]. While these efforts have helped to identify common methodologies to design and test systems, it is still unclear how to capitalize on the advantages of TUIs [13] [25].

Many researchers [10] [14] agree that TUIs may offer numerous advantages over GUIs. One advantage is the capability to retain knowledge through physical interaction with the TUI, allowing the system to potentially capitalize on users' motor learning capabilities. Motor learning is essential in the development of motor skills, from everyday tasks, such as walking, to advanced tasks like playing a piano [23]. TUIs can leverage motor learning for applications where recall is an essential process. One possible application domain is usable security, where researchers strive to create systems where passwords are easier to remember. If motor learning is leveraged properly, tangible password systems can be a possible alternative to alphanumeric password systems. Through repeated physical manipulation of the TUI, a user can store a password in their procedural memory [30]. This type of system can reduce the cognitive load placed on users who are often required to remember long and obscure passwords. 
This thesis presents an exploration of motor learning for tangible interaction by describing a novel tangible password system and a small study conducted to evaluate user performance while interacting with the system. This system will help indentify issues, which may arise while attempting to create a tangible password system that hopes to leverage motor learning. More generally, this work will act as an exploration of motor learning that may be applied to TUIs in other application domains.

\subsection{Organization}

The remainder of this paper is organized as follows: Chapter 2 will give an overview of related work; Chapter 3 will discuss a description and analysis of our novel tangible password system; Chapter 4 will describe the user study; Chapter 5 will discuss the findings; Chapter 6 will describe the conclusions, and future research objectives. 


\section{CHAPTER 2}

\section{Related Work}

\subsection{Usable Security}

The term usable security may be relatively unknown to individuals outside the realm of HCI. To try to understand, you can separate the two words and define them separately. To start, the International Organization for Standardization (ISO) has defined usability as "the effectiveness, efficiency and satisfaction with which users achieve specified goals in particular environments" [31]. The term can also be broken down into a quality measure with the following attributes: learnability, efficiency, memorability, errors, and satisfaction [19].

Security is defined by the Merriam-Webster dictionary as "the state of being free from danger or threat". This is a broad term and can be applied to different situations (e.g. financial security). More specifically, computer security has three main facets: confidentiality, integrity, and availability [3]. Using this information you can deduce that a usable security system is one that maintains the confidentiality, integrity and availability of information while also being easy to learn and remember, efficient, and satisfying to use. 
To achieve this goal, the HCI and Computer Security communities have worked closely together to develop systems that embody the above definition. From an HCI standpoint, many of these systems are designed to create passwords that are more memorable. Some examples are graphical, audio and icon based password systems.

\subsubsection{Graphical-Based Passwords}

Graphical passwords are a type of password where users are required to remember information related to an image or a set of images [29]. One popular implementation is the click-based graphical password method [27] [4]. In this system, a user is shown an image, or a set of images, and is required to click certain points on the image. If the user successfully clicks on the correct points, in the correct order, he or she is successfully authenticated to the system. Another popular implementation is the Passfaces method [20] [8]. Passfaces relies on a person's ability to recall a human face for an extended period. In this system, users are familiarized with a set of 3 to 7 distinct human faces. In order to authenticate to the system, users must pick their select set of faces from a wide range of faces shown on the screen. Both implementations rely on the picture superiority effect, which is a person's ability to remember images better then text [27].

\subsubsection{Audio-Based Passwords}

Audio-based password systems require users to select a set of audio clips to act as their password [11]. The user must then recall their password from a larger set of audio clips called distractors. A preliminary study based on the Musipass system [11] suggests audiobased passwords may be more memorable than traditional alphanumeric passwords. 


\subsubsection{Icon-Based Passwords}

Icon-based passwords (also called picture-based passwords) give users a set of distinct icons to act as their password [17]. In order to authenticate to the system, the user must either hit a key attached to their icon, or use the mouse to click on the icon from a grid of distractor icons. This concept also capitalizes on the picture superiority effect but is somewhat problematic since users may remember their set of icons but not the order in which they should be entered.

\subsection{Haptic and Gesture Password Systems}

Although there is no clear definition of what constitutes a tangible password system, closely related works include systems where haptic technology is used as the primary password identification mechanism, and gesture based systems where the gestures are performed with the input device, not on a screen.

\subsubsection{Haptic Password Systems}

Two systems that include the use of haptic technology are the Haptic Wheel [1] and the Secure Haptic Keypad [2], both of which are aimed at secure authentication in public places. In these systems, passwords are given in the form of a series of vibrotactile cues called tactons. For the Haptic Wheel, a user interacts with a rotary dial capable of continuous clockwise and counter-clockwise revolutions. The dial is fitted with two vibration motors that emit the vibrations. On top of the dial is a button, which allows users to select the current tacton. As the user rotates the dial, different tactons will be emitted 
through the dial and felt by the user. Each tacton is unique, and the location of tactons changes after each selection. This is done to prevent snoopers from observing the user's password entry and inferring their password based on their hand movements. The only visual aid to the user is the on screen interface that displays the number of PINs entered and the distance needed to reach the next adjacent tacton. Preliminary studies conducted to test the usability of the system show that users were able to distinguish tactons of different frequencies and were able to successfully enter their password for authentication.

For the Haptic Keypad, a user interacts with three keys capable of detecting touch and emitting tactons. For each password entry attempt, three different tactons are emitted (one for each key). Like the Haptic Wheel, the locations of the tactons are randomized after each user selection. However, unlike the Haptic Wheel which has the capability to emit more tactons, extra measures were taken with the Haptic Keypad to get a better understanding of the full potential of the system. To accomplish this, two different password entry modes were tested. The first is the normal input mode where a user selects the correct key that emits the correct tacton for his or her password sequence. The second is the hybrid mode where, before a key can be pressed, the system asks the user either to enter the correct password or simultaneously enter its compliment. In preliminary studies, users were successful in entering their passwords in both normal and hybrid mode, although mean authentication times were higher for the hybrid mode.

As both of these systems are aimed at authentication in public places, the randomization of the vibration patterns is vital as it protects the system from outside 
observation. However, this requirement does not give the user the ability to recall their password from constant physical interaction with the device. Instead, users must remember a haptic password the way they remember an alphanumeric password.

\subsubsection{Gesture Based Passwords}

Gesture based authentication systems, which require the user to perform gestures by physically manipulating a device, are often suitable for small mobile devices with accelerometers (e.g. smartphones and media players) [21]. GesturePIN [5] is a mobile authentication system that gives users the ability to generate passkeys by performing gestures on their mobile device. Gestures consist of a series of three-dimensional (3D) directional movements (e.g. tilt device left). A benefit of this system is the direct physical control of the gestures, making them easy for users to recall when they choose to generate a PIN. However, this system does not require the user to recall any specific series of gestures, allowing users to generate passkeys on the fly and requiring users to only recall a small subset of possible gestures.

\subsubsection{Summary}

Haptic and gesture based passwords offer some of the same advantages of TUIs, primarily the ability for the user to use his or her hands in a nonconventional keyboard/mouse interaction. According to Ishii, this should allow users to have a more direct interaction with the interface, increasing their effectiveness at performing their task. However, these applications do not take full advantage of the user's motor learning capabilities. The purpose of the proposed system is to design a tangible password system that allows users 
to utilize their motor learning capabilities in order to reduce cognitive load and utilize longer passwords.

\subsection{TUI Frameworks}

TUI frameworks are used to evaluate and compare tangible systems. Each framework consists of a set of criteria that can be used to categorize a tangible system on different levels. This gives researchers the opportunity to see where their system's strengths lie, and where their system can make improvements. This section will describe three popular TUI frameworks and taxonomies.

\subsubsection{Fishkin Taxonomy}

Fishkin's taxonomy [9] provides a measure of the tangibility of a system by analyzing a system using two measurements, embodiment and metaphor. Embodiment refers to the users understanding of how closely tied the system is to the tangible object they are manipulating. Embodiment has four levels, full, nearby, environmental, and distant. A full system is when the output device is the input device. Fishkin uses the example of a clay sculpture. When a user alters the clay on a sculpture, the output, or the result, is seen on the same sculpture. In this case, clay sculpting is described as full embodiment. A nearby system is when the output takes place near the input device. In this case, the output of the system has to be closely related, or directly coupled to the input. One example would be the bricks paradigm, where the output will be shown directly beneath the input devices. An environmental system is where the output is around the user. This typically occurs when 
tangible systems adjust the audio, light, or temperature levels in a space. In a distant system the output is said to be "over there", or on another screen or another room. Fishkin's uses a television remote as an example of distant embodiment.

The second measurement in the taxonomy, metaphor, is used in the TUI sense to mean, "Is the system effect of a user action analogous to the real-world effect of similar actions". The metaphor is broken down into two parts, shape and motion and five levels, none, noun, verb, noun and verb, and full. None refers to a system where no metaphor is employed. When a user performs an action with the object, the resulting output is not analogous to what would occur in the real world. A metaphor of noun refers to the shape, look, or sound of an object. This type of metaphor is usually implemented when the tangible object looks, feels, or sounds like a real-world object. A metaphor of verb is when performing an action with the object is similar to performing a similar action in the real world. A metaphor of noun and verb is when the noun and verb are tightly coupled to the point where performing an action with the tangible object is almost precisely like performing the same action with the same object in the real world. For example, in the Urp system [urp], blocks are used to represent buildings.. Arranging the blocks cast shadows on the landscape located beneath the objects. In this system, moving the blocks is like moving buildings in the real world. A full metaphor is one where no metaphor is needed since the virtual system is the physical system. This is similar to full embodiment where your action performed on the object results in exactly how it would in the real world (e.g. clay sculpting). 


\subsubsection{The TAC Paradigm}

The TAC (token and constraint) paradigm described by Shaer et. al. [24] is a unifying framework to describe the structure and functionality of TUIs. The paradigm is focused on the use of tokens and constraints. A token is a graspable physical object that represents digital information while a constraint is a physical object that limits the use and behavior of a token. A constraint can limit a token in three ways. A constraint can limit a token by its physical properties. This means the affordance of a constraint will inform the user how to manipulate a token. A constrain can also place limits on the physical interaction space of the token. Once the token has been placed within the confines of the constraint, the size of the constraint will limit the space of interaction. A constraint can also serve as a reference frame that limits the location of the token. The reference frame limits the token by informing the user the token must be within the confines of a certain area in order for the coupling to take place.

TAC has five properties, couple, relative definition, association, computational interpretation, and manipulation. Couple requires a physical object to be coupled with a variable (digital information) to be considered a token. For example, in the Marble Answering Machine, a marble with a message attached to it is considered a token, while a marble with no messages attached is not. Relative definition states that a physical object can be a token, a constraint or both. In the Marble Answering Machine, if the marbles are arranged in a queue, the marbles with messages are tokens, but since they constrain the movements of other marbles, they are also considered constraints. Association states that a 
TAC relationship is created when a token is associated with a constraint. When the token is removed from the constraint, this relationship is lost. Computational interpretation states that any manipulation of the token while in the constraint changes the state of the system while any manipulation done outside of the constraint has no effect on the system. The manipulation property states, that a token can be manipulated discretely, continuously, or a combination of the two.

\subsubsection{RBI Framework}

Reality-Based Interaction (RBI) is a framework used to describe post-wimp interaction [16]. Some examples of post-wimp interaction would be virtual reality, ubiquitous computing, and tangible interaction. This framework was created because the authors saw a common thread between many of the emerging interaction styles. These threads surrounded various themes of reality such as the "users' understanding of naïve physics, their own bodies, the surrounding environment, and other people". The authors used this commonality to create a framework based on four themes, naïve physics, body awareness and skills, environmental awareness and skills, and social awareness and skills.

Naïve physics (NP) is "the common sense knowledge about the physical world". This would include real-world properties such as gravity and friction. Body awareness and skills (BAS) is the "familiarity and understanding that people have of their own bodies..." This would include skills humans obtain over time such as the ability to walk or play an instrument. Environmental awareness and skills (EAS) is the ability for a person to understand their physical presence inside of an environment. This would include a 
person's spatial awareness. Social awareness and skills (SAS) draws from the fact that "people are generally aware of the presence of others and develop skills for social interaction". This would include a person's ability to pick on verbal and nonverbal communication cues.

Also included in the framework is a description of tradeoffs associated with having a form of reality in an interface. These tradeoffs are expressive power, efficiency, versatility, ergonomics, accessibility, and practicality. Expressive power is the user's ability to perform many actions with the same application. Efficiency is concerned with how quickly a user can perform tasks. Versatility is the system's ability to support task from different application domains. Ergonomics is concerned with how much stress or fatigue a user experiences while interacting with the device. Accessibility is concerned with users with varying levels of ability being able to use the system. Practicality refers to how practical the system is to develop, produce and deploy.

\subsubsection{Summary}

These frameworks provide three different ways to classify and compare tangible systems. Each of them is unique, giving a wide range of options for analyzing a system. Chapter 3 will describe how these frameworks were used to analyze the proposed tangible password system. 


\section{CHAPTER 3}

\section{TangibleRubik}

TangibleRubik is an attempt to create a novel tangible password system that takes advantage of a user's motor learning capabilities. This system is not intended to be used in practice. Instead, this system was created to further understand the advantages of such a system and to identify areas for improvement. It also acts as an example of how a motor learning for a tangible system can be applied to a specific application domain.

\subsection{Description}

TangibleRubik allows users to physically manipulate a Rubik's Cube for authentication to a system. A Rubik's Cube [22] is a 3D puzzle created by Ernő Rubik in 1974. Each face of the cube is divided into nine squares of equal size. The cube is constructed in such way that the individual rows and columns of each face are rotatable. The individual faces of the cube are also rotatable. This allows an individual to manipulate one or more of the rows, faces, or columns by performing a rotation. A user performing a rotation on one of the rows, columns or faces will be classified as a move. The various combinations of moves will act as 
the users' password. By having users physically manipulate the cube, the system aims to take advantage of humans' innate ability to recall motor actions through repetitions.

\subsection{Implementation}

TangibleRubik is implemented using a Rubik's cube ( $4 \times 4 \times 4$ inch) augmented with barcode like symbols called TopCodes [12]. These codes are detectable using a standard webcam and allow the system to determine the code's location and orientation. A code is placed on each block of the cube for a total of 54 distinct codes. Before each password entry attempt, the system must know the current location of the codes on each face. This is accomplished by a scanning process where the webcam takes a snapshot of each side of the cube. To do this, the user interacts with a simple graphical interface (see Figure 3.1). This interface was written in the Java programming language because the TopCodes library was created in Java. The interface provides the user with a frame showing the current feed from the webcam. This allows the user to see if the entire cube is within the webcams range and if the system is able to detect all of the codes on the current face. The user will hit the capture button to instruct the webcam to take a snapshot of the codes on the current face.

In order for the capture to be completed successfully, the faces must be entered in the same order every time. This protocol allows the system to create an internal representation of the cube's current state. The system carries out the user's password on the internal representation of the cube to determine the correct final cube state. If the protocol is not followed properly, the internal cube will not match the physical cube and any authentication attempt will result in a failure. 


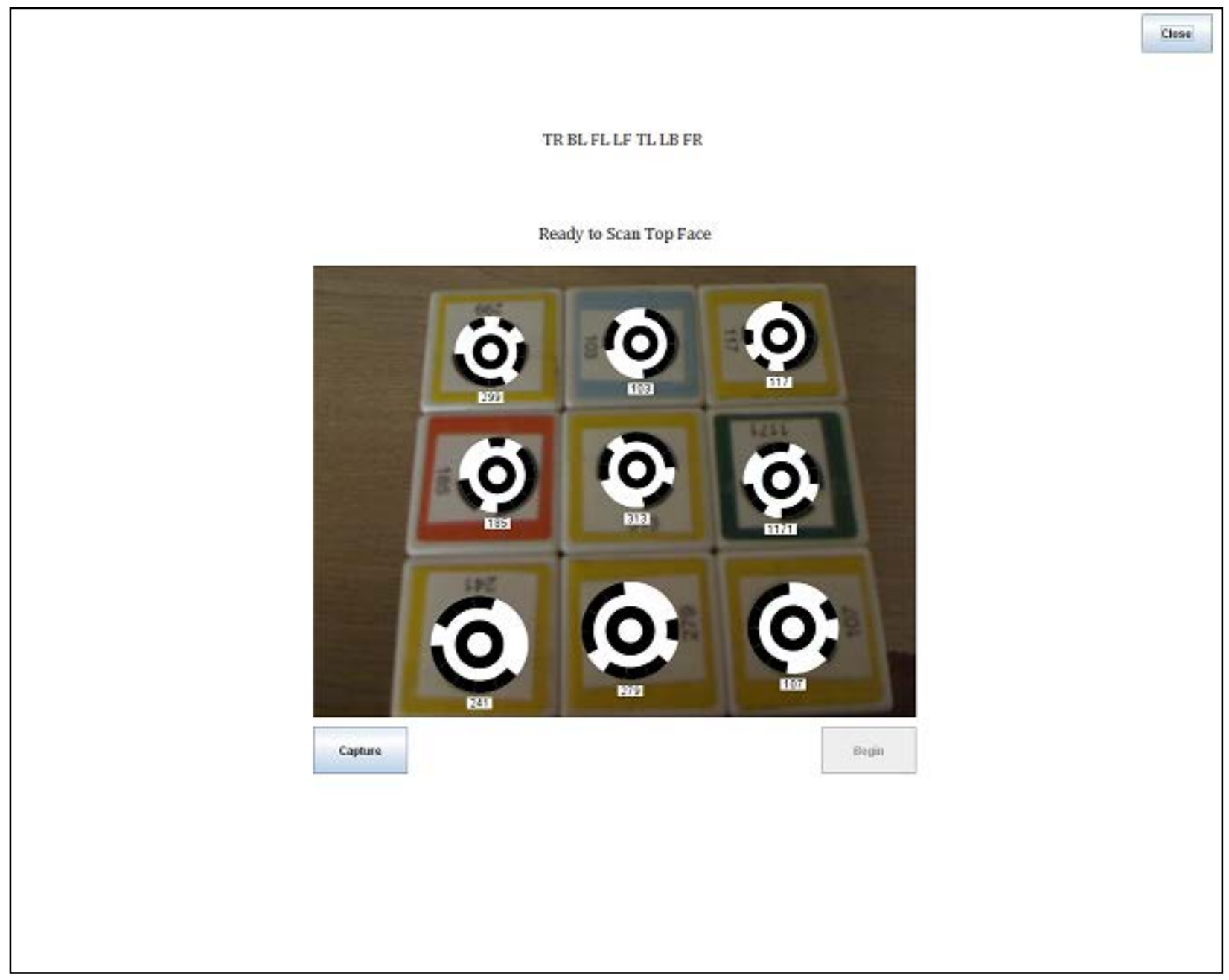

Figure 3.1: Simple GUI used for scanning during training

A user's password consists of a series of moves that can be performed on the cube. Passwords are given in the form of a sequence of letter pairs. The first letter represents which face of the cube to alter and the second represents the direction of rotation in relation to the user. Each face is given an orientation description (see Figure 3.2) and each move is given a rotation description (Right, Left, Front and Back). For example, the password, LB, BR and TL would instruct the user to first rotate the left face towards the 
back, then rotate the back face towards the right, and finally rotate the top face towards the left (or counter-clockwise). Users are limited to moves that alter the codes or the location of the codes on the top face (10 moves in total). The possible moves are top-left, top-right, front-right, front-left, back-left, back-right, left-front, left-back, right-front, and right-back.

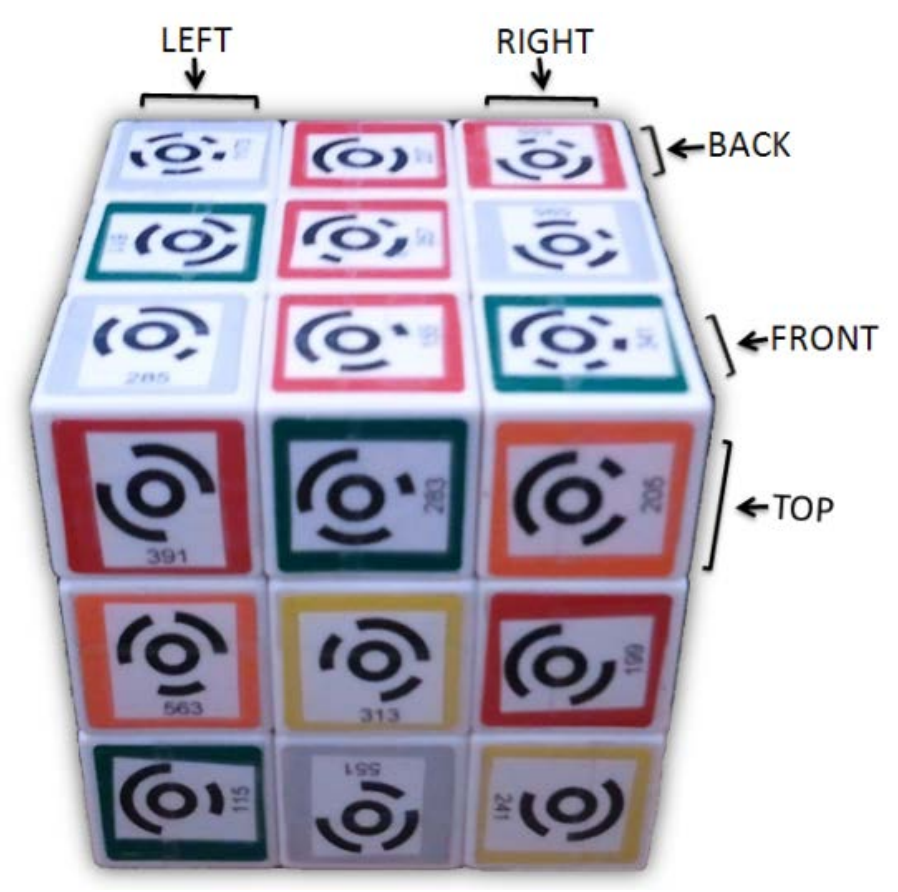

Figure 3.2: TangibleRubik, in orientaton held by participants, identifying the faces as in the passwords

The user must perform the moves in the order in which they are given, failure to do so will result in a failed authentication attempt. After performing their password, the user interacts with the graphical interface to scan the top face of the cube. The user must be sure to scan just the top face. If the codes present on the top of the user's cube matches with the internal cube, then the authentication was successful. 


\subsection{Analysis of TangibleRubik}

This section will give an analysis of TangibleRubik using the TUI frameworks and taxonomies discussed in Chapter 2. This analysis will provide an opportunity to see how TangibleRubik measures in its level of tangibility, and reality.

\subsubsection{TangibleRubik and the Fishkin Taxonomy}

Embodiment will be the first measurement used to analyze TangibleRubik. In this case, not just one of the classifications can be used. TangibleRubik is both full and distant. Full is appropriate because in the system, the user can refer to the Rubik's cube as their password. By performing moves on the cube (the input), the user is transforming the cube into their correct password (the output). At the same time, the system has characteristics of a distant system since the user must use the webcam and the graphical interface to scan the cube and to enter the password.

Attempting to classify TangibleRubik using one of the metaphor descriptions is a difficult task. This is due to the abstract nature of passwords. Passwords do not have an inherent real-world meaning. If a password was a real-world object what would it be? The inability to classify TangibleRubik using one of the metaphor descriptions described may be due to the author's inability to foresee tangible systems manipulating such an abstract concept. A more up to date description should include a classification where the description of the object matches the description of the manipulated data. For example, a 
password may be described as something that is difficult to crack or hard to figure out. You may get a similar response if one was asked to describe a Rubik's cube.

\subsubsection{TangibleRubik and TAC}

To use TAC to describe TangibleRubik the tokens and constraints must be identified. In this regard, the Rubik's cube is classified as a token and the webcam as a constraint. The cube must be the token since it is the physical object that is coupled with the digital information (the password). The webcam acts as a constraint since the scanning process and the password entry must be performed using the webcam. In this case, the webcam is serving as a reference frame, limiting the placement of the cube.

Concerning the properties of TAC, the coupling property has already been established since the cube is coupled with the user's password. Relative definition is not very relevant in our case since the cube only acts as token and the webcam only acts as a constraint. Association is also not very relevant since the TAC is formed once the user begins to scan the cube. Before this occurs, the cube is just a cube and the webcam just a webcam. The association process is when then tangible system is created. The most intriguing and hard to classify definition is the computational interpretation. According to TAC, the token must always be in the constraint for the system to change states. In this case, this is partly true since the scanning and authentication processes occur when the cube is beneath the webcam. On the other hand, the user does not need to keep the cube under the constraints of the webcam to alter its state. The token is free to be manipulated anywhere the user chooses. The manipulation definition can be considered both discrete 
and continuous since a user may make a series of discrete moves or one continuous action that counts as two or more moves (performing two subsequent left-front moves can be done in one continuous action).

\subsubsection{TangibleRubik and RBI}

In using the themes of RBI to analyze TangibleRubik, it seems appropriate not to discuss SAS and EAS since their descriptions do not have much impact on the system. However, TangibleRubik relies heavily on NP and BAS. In TangibleRubik, users manipulate the cube knowing that performing a move on the cube will result in a direct change to the cube's state. The user knows this from his or her previous experience with other physical objects. The same may not be true if the user was given a virtual representation of the cube, since he or she would have to determine what interface controls resulted in the move he or she wishes to perform. In this regard, NP helps remove a level of abstraction between the user and the task he or she wants to perform, in turn, making the interface "closer to the user. TangibleRubik also draws upon BAS by relying on the user's ability to recall actions through repetition. By leveraging motor learning to reduce cognitive load, the system takes advantage of the physical skills already possessed by the user.

The RBI tradeoffs show what the system gives up in order to gain more reality. Expressive power and versatility have been sacrificed since the system is highly specialized. Although it is possible to create another tangible system that utilizes a Rubik's cube and a webcam, the current system only supports one function, which is password entry. Efficiency may be an issue for users with poor dexterity when first interacting with 
the system. Since most users may not interact with a Rubik's cube in their day to day life, efficiency may be a problem when first learning how to use the system. Ergonomics should not be an issue since manipulating the cube should not cause must stress or fatigue. Accessibility may be a problem for users who suffer from motor impairments, arthritis, or loss of limbs. Practicality may also be an issue since the system would require users to purchase one or two additional pieces of equipment.

\subsubsection{Summary}

Analyzing TangibleRubik using these frameworks and taxonomies gives a better understanding of the system's strengths and weaknesses. It also shows areas where current frameworks are lacking and may need revision or improvement. As TangibleRubik is a novel attempt to explore motor learning in a specific application domain, future revisions of the system may alter how the system measures in these three cases. 


\section{CHAPTER 4}

\section{Method}

\subsection{Introduction and Predictions}

This chapter will describe the study conducted to test the effectiveness of TangibleRubik. The primary goal of this study was to determine if users could use the system to accurately and quickly enter their password. A second goal was to determine what effect differing password lengths would have on a user's ability to recall their password after being removed from the system for a brief period. The hypothesis is users given passwords of longer length will have a higher error rate during both training and experimental trials, as well as a higher fail rate on final authentication attempts. As such, this study is not a comparative study of TangibleRubik vs. other types of passwords. Nor does it have the goal of demonstrating the efficacy of the TangibleRubik system. Rather, this study is a proof of concept to determine if tangible passwords systems could be a viable alternative to more traditional password systems. 


\subsection{Password Creation}

The sequences of moves, which make up the participants passwords, were randomly generated. The passwords were created by a small program that randomly generated numbers, which corresponded to a move. Some passwords were discarded if two consecutive moves resulted in a null move. That is, if a move is the direct opposite of the preceding move, that would result in a null move. For example, the password TL, FR, FL, LB, RF, LB, TR would be an invalid password since the user consecutively performs a FR and FL move. This is considered a null move since the user could choose not to perform these rotations since the ending position will be the same as the start position. The same may not be true if the moves are separated by one or more moves.

\subsection{Physical Setup}

The study was performed at Bowling Green State University in the Computer-Human Interaction Lab (CHIL), located at 227 Hayes Hall. Participants were seated in front of the workstation and were given a standard mouse in order to interact with the GUI. A base, created from Lego ${ }^{\mathrm{TM}}$ blocks, was placed in front of the user. The base served as the resting place for TangibleRubik during any scanning process. The base was taped to the desk to ensure that a subject would not accidently displace it. A Logitech webcam was used to capture the topcodes. A Rubik's Twist was used to create an apparatus for the webcam to hang from. The webcam pointed down over the base in order to capture the topcodes at a reasonable distance. 


\subsection{Subjects}

Eleven college student participants ( 7 men, 4 women) with a mean age of 22 volunteered for this study. All participants reported they had some experience with tangible interfaces (e.g. tablets, smartphones, Nintendo Wii remote, etc.).

\subsection{Experimenters}

The author was the sole experimenter. An experimental script was read to each subject. This was done in order to limit any bias and to ensure the experiment was run smoothly. The author completed the required HSRB training before running any participants.

\subsection{Experimental Design}

The study tested two conditions using a between subjects design. Six participants in the first condition were given a seven-move password and five participants in the second were given a 10-move password. The independent variable was password length (either 7 or 10 moves). The dependent variables were time (measured in seconds) and success (either a successful or unsuccessful authentication attempt). Time and success were vital measures since they provided a way to determine how long it took participants to enter their password, and how many successful and unsuccessful attempts were made. 


\subsubsection{Demographics}

Before the experiment began all participants were asked to fill out a demographics form. The information collected from the form was age, sex, experience with tangible interfaces, experience with graphical based authentication systems, and handedness.

\subsubsection{Practice and Experimental Trials}

All data from the practice and experimental trials were collected from the Java program driving the experiment. The information collected was number of successful/unsuccessful authentication attempts, and authentication time.

\subsubsection{TLX Questionnaire}

After the participants completed their experimental trials, they were asked to fill out a NASA Task Load Index [18]. This questionnaire measures the workload placed on the participant during the experiment on six different categories. The six categories are mental demand, physical demand, temporal demand, performance, effort, and frustration.

\subsection{Procedure}

Once the participants arrived to the lab they were asked to read and sign a consent form. The participants then filled out the questionnaire sheet. While the participant filled out the questionnaire, the experimenter assigned the participant to one of the two password groups. In particular, the participant was assigned to the opposite group of the previous participant. This was done to ensure that the groups were equally distributed. In order to familiarize the participant with his or her password, and the system, each participant 
completed a training session. The experimenter gave an overview of TangibleRubik and gave a demonstration of all possible moves. Participants were told how to use the GUI to scan During the training session each participant was required to successfully enter their password three consecutive times before moving on to the experimental trials. Textual representations of the passwords were visible to the user at all times during the training trials. This was done to aid in the learning of the password. Before the participant began the training sessions, the experimenter explained the password to the user and gave a demonstration of how the password should be completed. The experimenter was able to answer any questions the participant may have during the training trials. Most questions asked about clarification on move descriptions.

Experimental trials directly followed training and required participants to correctly enter their password five consecutive times. This objective criterion was chosen to ensure that all participants had achieved the same level of competency, by the end of the experimental trials, in storing their password to memory. The participant's password was not present during the experimental trials. However, if a participant had three consecutive failed authentication attempts, the experimenter reminded the participant of his or her password. Besides reminding the participant of their password after three failed authentication attempts, the experimenter was not able to answer any questions.

After the experimental trials, participants were given a distractor task, which consisted of a word search, to mentally remove them from experimental task for a duration 
of 10 minutes. After the distractor task, participants were asked to enter their password a final time as test of password mastery. 


\section{CHAPTER 5}

\section{Results and Analysis}

\subsection{Results}

This chapter reports the results of the study and provides an analysis of the data.

\subsubsection{Impact of password lengths}

Hypothesis - participants given passwords of longer length will have a higher error rate during both training and experimental trials, as well as a higher fail rate on final authentication attempts.

Comparing between the two password length conditions, no significant differences were found between the error rates in either the training and experimental trials, as shown in Figure 5.1. Similarly, performance on normalized (by password length) time-per-move on the final authentication attempt was not significant by condition. 


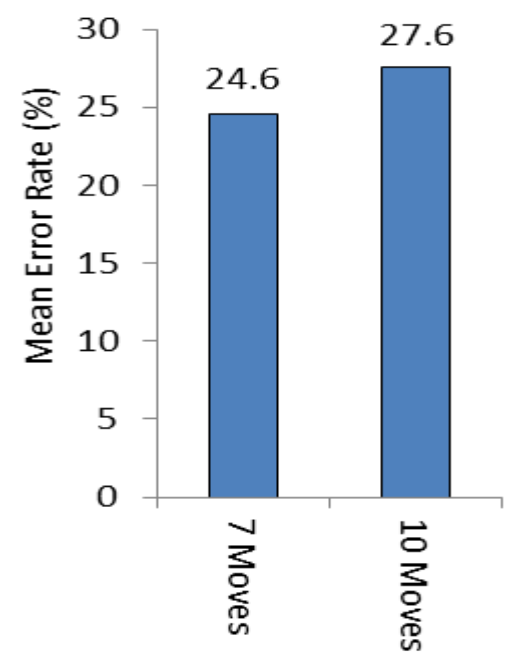

Figure 5.1: Mean error rate

It should be noted that on the final authentication attempt, all participants were able to successfully enter their password after the distractor task. Mean password entry times for the two experimental conditions (see Figure 5.2) were not significant by condition, likely due to high variance.

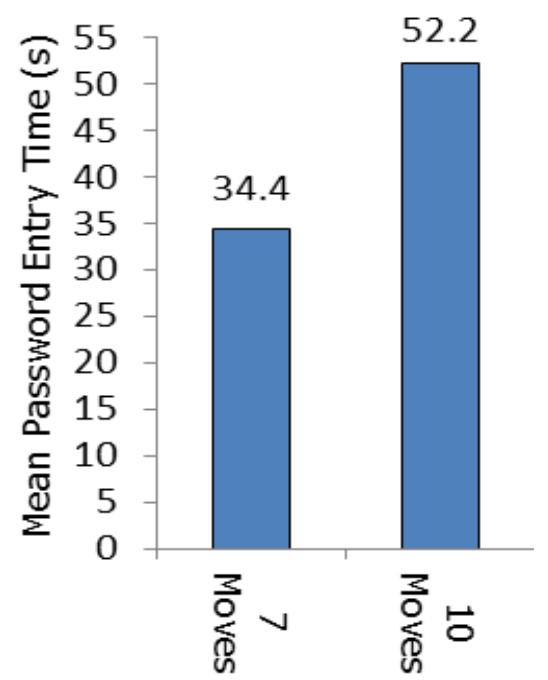

Figure 5.2: Mean password entry times 


\subsubsection{TLX Questionnaire}

No significant differences were found between groups in the TLX questionnaire data.

However, correlation data revealed a significant positive correlation between both physical demand $(R(9)=0.74, p<.05)$ and frustration ratings $(R(9)=0.74, p<.05)$ with the normalized time-per-move on the final authentication attempt. A summary of the TLX data is given in figure 5.3 .

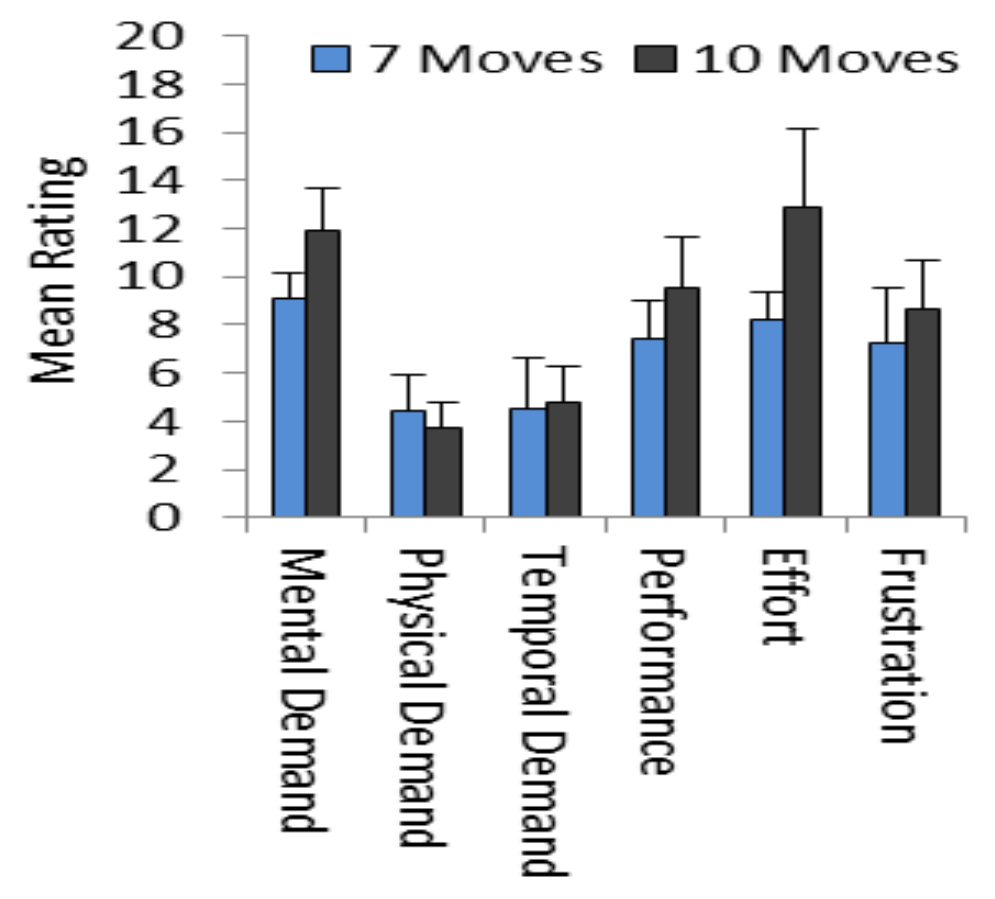

Figure 5.3: Summary of the results from the NASA TLX 


\subsection{Analysis}

\subsubsection{Analysis of Error Rates}

Error rates during the experimental trials were surprising due to the prediction that participants in the 10-move condition would experience significantly higher error rates than those in the 7-move condition. This indicates that participants were able to utilize longer passwords just as effectively as users with shorter passwords. Although error rates are higher than hoped, this may be an artifact of participants adjusting to a new system. Given that all participants were able to recall their password after performing the distractor task, this suggests participants were successful in storing their password in memory (at least for the short term). This result is promising as it shows users have the capability to learn and recall passwords of considerable length.

\subsubsection{Analysis of Password Entry Times}

Password entry times for participants in the 10-move condition were higher on average. However, the high level of variance suggests that longer entry times were a result of individual differences (e.g. previous Rubik's cube experience, dexterity issues) rather than password length. Although password entry times were not significant by condition, the correlation between frustration, physical demand and the normalized time-per-move suggest TUIs, which require users to perform task they find to be physically demanding, can have a cascading effect, leading to higher frustration levels. 


\section{CHAPTER 6}

\section{Discussion and Conclusion}

Since leveraging motor learning for tangible interaction is relatively unexplored, this system provided a basis to understand what must change in order for such a system to be used in practice. The exploratory study provided useful information that identified items that must be addressed, and will determine future research objects.

\subsection{Discussion of Password Entry Times}

Mean entry times of 34 and 52 seconds are too long and must be shortened before they can be used in practice as measured against a traditional alphanumeric password system. The use of tangibles for password systems may be at an inherent disadvantage in terms of entry times when compared to alphanumeric systems, since most users will have many years of experience with a keyboard and only minutes with the tangible object. The Haptic Keypad system discussed earlier had reported password entry times of 22.2 and 33.8 seconds for their 6 and 9 pin password conditions. The Haptic Wheel study provided similar results with authentication times of 23.0 and 23.5 seconds for the 6 pin and 9 pin conditions. However, since the Haptic Keypad and Haptic Wheel systems are designed for secure 
authentication in public places, a user may be willing to give up the extra seconds it takes for authentication if they are convinced no one is recording their password.

\subsection{Discussion of Password Memorability}

A system which leverages motor learning may offer its own benefits (e.g. memorability), but at this time it is unknown exactly how memorable the system's passwords are. Although subjects were able to recall their password after being removed from the system for ten minutes, subjects should be able to remember their password for weeks and months. To address this, a longitudinal study, which follows the user's ability to recall their password over weeks, may provide a more concise picture of the memorability of the system. In addition, a study where the memorability of alphanumeric and tangible passwords is compared may strengthen the argument that tangible systems can be more memorable. Another possible study can look at how the passwords were given to the users. In this case, passwords were given as a textual representation. If the passwords were given as an animation or another form, users may have made different mechanisms to remember their passwords. This study can also explore if the memorability of the textual representations of the tangible passwords are as memorable as the tangible equivalent. It is important to remember that this study did not set out to prove that tangible passwords are more memorable, but to test if users would be able to use a tangible system centered on motor learning. 


\subsection{Discussion of Password Length}

In this study, the passwords were seven and ten moves long. In terms of the memorability and entry times of the passwords, shorter or longer length passwords will most likely produce different results. A separate study, looking at the differing lengths of tangible passwords, may provide some more information on how long tangible passwords can be before user performance begins to deteriorate. A more in depth analysis of how many moves would be suitable for a tangible password should also be discussed.

\subsection{TangibleRubik and Password-Composition Policies}

One area of research, which may produce interesting results, would be to analyze the relationship of tangible passwords and password-composition policies. Passwordcomposition policies are the rules that govern what characters a user's password can and must include [Komanduri]. For example, a password-composition policy for a university's email system may require users to have a special character (e.g. @, \&, \$), an uppercase letter, and at least one number. This is done to increase the security of the user's password from brute-force and dictionary based attacks. This policy also discourages users from choosing predictable passwords such as their name and birthday. A tangible system, similar to TangibleRubik, may not be subject to the same constraints since the moves have no inherent meaning. This may be a benefit users are willing to sacrifice password entry time for, especially if users can use the same tangible password for multiple entities. This is 
often an issue with alphanumeric password systems since different entities may have different policies, requiring the user to remember several passwords.

\subsection{User Created Passwords}

For this study, subjects were not able to construct their own passwords. An important analysis of tangible password systems must include a study where users are able to create their own password. This research will give us a better understanding of how memorable tangible passwords can be if users create them for themselves. This will also provide a way to analyze what simple passwords users create. It may be the case that users create passwords with very few moves (e.g. RB, LB, RB, LB, RB, LB, RB). This type of information can be critical for a future practical tangible password system. These results could argue the need for tangible password systems to be governed by password-composition policies.

\subsection{Conclusion}

On a micro level, this thesis has presented an early examination of an exploratory tangible password system. The goal of this research was to understand how a tangible password system could leverage motor learning in order to reduce cognitive load placed on users and allow them to utilize longer passwords. A preliminary study shows that users can recall seven and ten length passwords after being removed from the system for a short time. These results indicate that tangible passwords, which leverage motor learning, may have the capability to become a suitable alternative to alphanumeric systems. More research is 
needed by the HCI and Security communities to determine how this type of tangible password system can be better utilized in practice.

On a macro level, the goal of this research was to understand how to capitalize on the advantages of TUIs in a particular application domain. The hope is to take TUIs out of the research laboratories and bring them into the homes and offices of everyday computer users. This work provided a basis for future work to continue to investigate how this can, and should be done. 


\section{BIBLIOGRAPHY}

[1] A. Bianchi, J.K. Lee, I. Oakley, and D.S. Kwon. The Haptic wheel: Design \& Evaluation of a Tactile Password System. In Extended Abstracts of CHI 2010, pages 3625-3630, ACM, 2010.

[2] A. Bianchi, I. Oakley, and D.S. Kwon. The Secure Haptic Keypad: Design and Evaluation of Tactile Password System. In Proceedings of the 2010 SIGCHI conference on Human factors in computing systems, pages 1089 - 1092, ACM, 2010.

[3] M. Bishop. Computer Security. Upper Saddle River. Pearson Education, Inc. 2003. Print.

[4] S. Chiasson, A. Forget, E. Stobert, P.C. Oorschot, and R. Biddle. In Proceedings of the $16^{\text {th }}$ ACM conference on Computer and communications security, pages $500-511$, ACM, 2009.

[5] M.K. Chong, G. Marsden, and H. Gellerson. GesturePIN: using discrete gestures for associating mobile devices. In Proceedings of SIGCHI's International Conference on Human-Computer Interaction with Mobile Devices and Services, pages 261 - 264, ACM, 2010. 
[6] M. Coelho, J. Zigelbaum, and J. Kopin. Six-forty by four-eighty: the post-industrial design of computational materials, In Proceedings of the fifth international conference on Tangible, embedded, and embodied interaction, pages 253 - 256, ACM. 2011.

[7] E. Costanza, M. Giaccone, O. Kung, S. Shelly, and J. Huang. Tangible Interfaces for Download: Initial Observations from Users' Everyday Environments, In Proceedings of the 2010 SIGCHI conference on Human factors in computing systems, pages 2765 2774, ACM, 2010.

[8] P. Dunphy, J. Nicholson, and P. Oliver. Securing Passfaces for Description, In Proceedings of the $4^{\text {th }}$ symposium on Usable privacy and security, ACM, 2008.

[9] K.P. Fishkin. A taxonomy for and analysis of tangible interfaces. In Ubiquitous Computing, pages 347 - 358, Springer-Verlag, 2004.

[10] G.W. Fitzmaurice, H. Ishii, and W. Buxton. Bricks: Laying the Foundation for Graspable User Interfaces, In Proceedings of the 1995 SIGCHI conference on Human factors in computing systems, pages 442-449, ACM, 1995.

[11] M. Gibson, K. Renaud, M. Conrad, and C. Maple, Musipass: authenticating me softly with "my" song, In Proceedings of the 2009 workshop on New security paradigms workshop, pages 85 - 100, ACM, 2009.

[12] M. Horn. TopCode: Tangible Object Placement Codes, http://users.eeces.northwestern.edu/ mhorn/topcodes/ 
[13] E. Hornecker, R. Jacob, C. Hummels, B. Ullmer, A. Schmidt, E. van den Hoven, and A. Mazalek. TEI goes on: Tangible and embedded interaction. IEEE Pervasive Computing Magazine/Journal, 7(2): 91-95, 2008.

[14] H. Ishii and B. Ullmer. Tangible Bits: Towards Seamless Interfaces between People, Bits and Atoms. In Proceedings of the 1997 SIGCHI conference on Human factors in computing systems, pages $234-241$, ACM, 1997.

[15] J.H. Israel, O. Belaifa, A. Gispen, and R. Stark. An Object-centric Interaction Framework for Tangible Interfaces in Virtual Environments. In Proceedings of the fifth international conference on Tangible, embedded, and embodied interaction, pages 325-332, ACM, 2011.

[16] R.J. Jacob, A. Girouard, L.M. Hirshfield, M.S. Horn, O. Shaer, E.T. Solovey, and J. Zigelbaum. Reality-Based Interaction: A Framework for Post-WIMP Interfaces, In Proceedings of the 2008 SIGCHI conference on Human factors in computing systems, pages 201- 2010, ACM, 2008.

[17] S. Komanduri and D.R. Hutchings. In Proceedings of Graphics Interfaces 2008, pages 115 - 122, Canadian Information Processing Society, 2008.

[18] NASA TLX: Task Load Index. http://human-factors.arc.nasa.gov/groups/TLX/

[19] J. Nielson. Usability Engineering. San Francisco. Morgan Kaufmann. 1993. Print.

[20] Passfaces: http://www.realuser.com/ 
[21] S.N. Patel, J.S. Pierce, and G.D. Abowd. A gesture-based authentication scheme for untrusted public terminals. In Proceedings of the $17^{\text {th }}$ annual ACM symposium on User interface software and technology, pages 157 - 160, ACM, 2004.

[22] Rubik's Cube: http://www.rubiks.com/

[23] R.A. Schmidt, A Schema Theory for Discrete Motor Skill Learning. Psychological Review, 82(4): 225 - 260, 1975.

[24] O. Shaer and R. Jacob. A specification paradigm for the design and implementation of tangible user interfaces. Transactions on Computer-Human Interaction, 16(4), ACM, 2009.

[25] O. Shaer and E. Hornecker. Tangible User Interfaces: Past, Present, and Future Directions, Foundations and Trends in Human-Computer Interaction, 3(1-2), Now Publishing, 2010.

[26] G.C. Smith. The Hand That Rocks the Cradle. I.D. May/June, pages $60-65$.

[27] E. Stobert. Usability and Strength in Click-based Graphical Passwords. In Proceedings of the $28^{\text {th }}$ international conference extended abstracts on Human factors in computing systems, pages 4303 - 4308, ACM, 2010.

[28] J. Underkoffler and H. Ishii. Urp: a luminous-tangible workbench for urban planning and design. In Proceedings of the SIGCHI conference on Human factors in computing systems, pages 386 - 393, ACM, 1999. 
[29] S. Wiedenbeck, J. Waters, L. Sobrado, and J.C. Birget. Design and Evaluation of a Shoulder-Surfing Resistant Graphical Password Scheme, In Proceedings on the working conference on Advanced Visual Interfaces, pages 177 - 184, ACM, 2006.

[30] D.B. Willingham, M.J. Nissen, and P. Bullemer. On the Development of Procedural Knowledge. Journal of Experimental Psychology: Learning, Memory, and Cognition, 15(6): 1047 - 1060. American Psychological Association, 1989.

[31] World Wide Web Consortium (W3C): Usability - ISO 9241 definition. http://www.w3.org/2002/Talks/0104-usabilityprocess/slide3-0.html

[32] A. Wu, D. Reily, A. Tang, and A. Mazalek. Tangible navigation and object manipulation in virtual environments, In Proceedings of the fifth international conference on Tangible, embedded, and embodied interaction, pages 37 - 44, ACM, 2011. 


\title{
Appendix A: Consent Form
}

\author{
BGSU。 \\ DOWLING GREEN STATE UNIVERSITY \\ Department of Computer Science \\ Consent to Participate in a Research Project \\ Bowling Green State University \\ Bowling Green, $\mathrm{OH} 43403$
}

Hello. You are invited to participate in a research project about the effectiveness of a tangible authentication system. A tangible authentication system is an authentication system which requires users to use their hands to manipulate : physical object in order to input a password to a computing device. As part of our work on this project in the Department of Computer Seience, we are conducting a study to help us understand how motor learning, or muscle memory, can be leveraged to improve the effectiveness of tangible user interfaces. In our research project, you will be asked to complete several task using a novel tangible authentication system. You will also be asked to complete a questionnaire regarding your previous experience with tangible interfaces and to rate your experience after the experiment is completed.

You will be asked to participate in one session which will last no more than 1 hour and 30 minutes; most sessions will last no more than one hour.

The anticipated risks to you are not greater than those normally encountered in daily life.

The benefit to this project is that we can leam how to better design tangible user interfaces so that users can have a more enjoyable computing experience while using this technology.

Information that you provide will remain confidential and your identity will not be revealed. Confidentiality of you as a respondent and your responses will be protected throughout the study and publication of study results. All of your responses will remain locked in a secure area within Hayes Hall. Your identity will not be revealed in any published results.

Your participation in this study is completely voluntary, and you can refrain from answering any or all questions without penalty. If your instructor has agreed to provide extra credit for your participation in this study your instructor will be notified after the conclusion of the experiment. If you decide to participate and change your mind later, you may withdraw your consent and stop your participation at any time without penalty. Your participation will have no impact on your compensation, your grades, class standing of relationship to the institution. Again, you are free to withdraw consent and to discontinue participation in the project at any time.

If these conditions have been explained to you, you are willing to participate, you have had your questions answered, and you are age 18 or over, please sign below

If you have any questions or comments about this study, you can contact us Martez. E. Mott or G. Michąel Poor, Visiting Assistant Professor Computer Scienee Department Bcowling Gireen State University Bowling Green, OH 43403 419-372-2337 memoti@bsa.di

You may also contact the Chair, Human Subjects Review Board if any problems or concems arise during the course of the study Bowling Green State University (419) $372-7716$ hishiabonect bissu odu 


\section{Appendix B: Demographics}

Participant \#:

Group:

\section{General}

1. Age:

2. Sex:

Male

Female

s. Do you have experience with tangible interfaces (tablets, touch screen devices, Nintendo Wii remote, etc.)?

Yes No

4. If Yes:

a. How much experience would you say that you have had with tangible interfaces? (1-Not much, 5-A great deal)

1

2

3

4

5

5. Have you ever used a graphical based authentication system?

Yes

No

6. Which hand is dominant?
Left
Right
Neither (ambidextrous) 


\section{Appendix C: NASA TLX}

Figure 8.6

\section{NASA Task Load Index}

Hart and Stavelands NASA Task Load index (TLX) method assesses work load on fwe 7-poirt scales. Increments of high, medium and low estimates for each point result in 21 gradations on the scales.

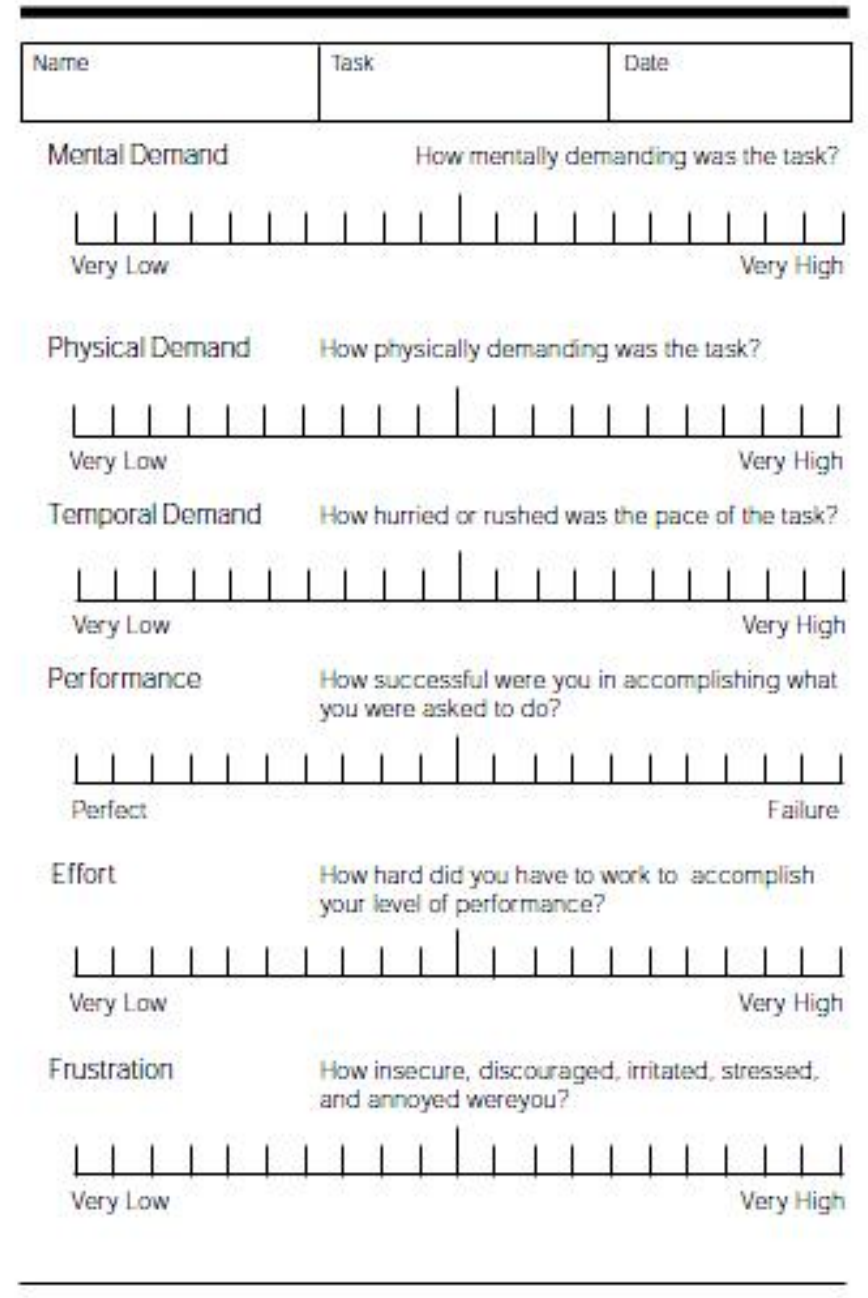

